\title{
Diversidad y cobertura de la comunidad bentónica del arrecife Enmedio, Sistema Arrecifal Lobos-Tuxpan, México
}

\section{Diversity and coverage of the benthic community at Enmedio reef, Lobos-Tuxpan Reef System, Mexico}

\author{
Marlene González-González ${ }^{1}$,Vicencio de la Cruz-Francisco ${ }^{1 *}$, Itzel Morales-Quijano ${ }^{1}$ y Rosa \\ Estela Orduña-Medrano ${ }^{1}$
}

\begin{abstract}
RESUMEN
La cobertura bentónica es un atributo importante para gestionar eficientemente los arrecifes coralinos, sin embargo, muchos ecosistemas marinos de Veracruz aún carecen de esta información y el arrecife Enmedio es un claro ejemplo; por esta razón, se estimó la diversidad y cobertura bentónica en el arrecife frontal, arrecife posterior y laguna arrecifal, utilizando cuadrantes de $1 \mathrm{~m}^{2}$, los cuales fueron colocados a cada $5 \mathrm{~m}$ sobre tres transectos de $50 \mathrm{~m}$ de largo. Con los datos recabados se estimaron la riqueza específica, equitatividad, dominancia de Simpson, diversidad de Shannon y diversidad verdadera, asimismo, se determinaron diferencias significativas mediante análisis de similitud y ordenación. La cobertura biótica promedio estimada para el arrecife fue de 37.3\%, principalmente se destacan los corales dado que aportaron la mayor cobertura con $19.8 \%( \pm 18.8)$. Las especies más importantes en cobertura fueron: Orbicella annularis, Erytropodium caribaeorum, Colpophyllia natans y Pseudodiploria clivosa. Los ambientes arrecifales son significativamente diferentes en composición y cobertura biótica con base en las pruebas de similitud y ordenamiento, ya que el arrecife posterior registró un mayor número de especies y de cobertura, básicamente por corales masivos; en cambio en la planicie arrecifal se determinaron varias zonaciones bentónicas que tienden a ser equitativas y con mayor número de especies efectivas, mientras en el arrecife frontal decrecen la diversidad, la cobertura y la equitatividad debido a que predomina el pavimento. Estos contrastes registrados en el arrecife Enmedio en la cobertura por especie y por ambiente arrecifal parecen ser normales dado que también han sido reportados para varios arrecifes del SAV.
\end{abstract}

Palabras claves: Corales, octocorales, diversidad, cobertura, arrecife Enmedio.

\begin{abstract}
Benthic coverage is an important attribute for the efficient management of coral reefs. However, many marine ecosystems of Veracruz still lack this information and Enmedio reef is a clear example. For this reason, the benthic diversity and benthic coverage were estimated on the front reef, back reef and reef lagoon. One $\mathrm{m}^{2}$ quadrants were used and placed every $5 \mathrm{~m}$ on 3 transects of $50 \mathrm{~m}$ long. The species richness, evenness, Simpson index, Shannon's diversity index and true diversity were estimated with the data collected. In addition, significant differences were determined using similarity and ordination analysis. The average biotic coverage estimated for the reef was $37.3 \%$;
\end{abstract}

\footnotetext{
Facultad de Ciencias Biológicas y Agropecuarias, Campus Tuxpan, Universidad Veracruzana. Carr. Tuxpan-Tampico km 7.5, Col. Universitaria CP. 92860, Tuxpan, Veracruz, México. marleneglez1392@gmail.com; delacruzf17@ hotmail.com*; quijanobm93@hotmail.com; aletse_o@hotmail.com
}

Recibido: 2 de marzo de 2016

Corregido: 6 de junio de 2016

Aceptado: 10 de junio de 2016

DOI: http://dx.doi.org/10.15359/revmar.8-2.3 
mainly corals stand out with the most coverage, 19.8\% $( \pm 18.8)$. The most important species in coverage were: Orbicella annularis, Erytropodium caribaeorum, Colpophyllia natans and Pseudodiploria clivosa. Reef environments are significantly different regarding biotic composition and coverage based on similarity and ordination analysis. For instance, a greater number of species and coverage, basically massive corals, were recorded in the back reef, while several benthic areas were found in the reef flatland, with a tendency to be more equitable and with a greater number of effective species. Finally, diversity, coverage and evenness decrease in the front reef due to the predominant presence of pavement. These differences registered in Enmedio reef regarding coverage by species and reef environment seem to be normal since they have also been reported for several other reefs from the Veracruz reef system.

Keywords: Corals, octocorals, diversity, coverage, Enmedio reef.

\section{INTRODUCCIÓN}

La cobertura bentónica en arrecifes coralinos es un atributo importante, dado que es un componente que explica cambios en las comunidades y valora el estado de conservación o deterioro de un arrecife coralino ante eventos naturales y por factores de origen humano, siendo estos últimos los que más deterioro están causando en los arrecifes de coral, principalmente los que están cercanos a la costa son los más vulnerables (Burke et al. 2011) ante perturbaciones por tránsito marítimo, contaminación, sobrepesca y turismo (Horta-Puga, 2010). Sin embargo, la información que se destaca en los arrecifes de Veracruz son fundamentalmente inventarios taxonómicos y descripciones que detallan la zonación arrecifal (Chávez et al. 1970; Quintana, 1991; HortaPuga \& Carricart-Ganivet, 1993; Jordán-Dahlgren, 1993; Beltrán-Torres \& Carricart-Ganivet, 1999; JordánDahlgren \& Rodríguez-Martínez, 2003; Chávez et al. 2010; Ortiz-Lozano et al. 2013), mientras la cobertura bentónica ha sido investigada especialmente en el Sistema Arrecifal Veracruzano (SAV) (Jones et al. 2008; Horta-Puga \& Tello-
Musi, 2009; Horta-Puga et al. 2015; Pérez-España et al. 2015); con esta información ha sido posible conocer la estructura comunitaria de este sistema arrecifal y plantear estrategias de manejo, con el fin de minimizar el impacto que genera el desarrollo costero del puerto de Veracruz.

Por el contrario, en el Sistema Arrecifal Lobos-Tuxpan (SALT), la riqueza taxonómica de algas, esponjas, corales y octocorales ha sido la principal información generada sobre comunidades bentónicas sésiles (Chamberlain, 1966; Chávez et al. 1970; Jordán-Dahlgren, 2002; González-Gándara et al. 2007; 2009; 2015); en cuanto a la cobertura del bentos arrecifal no es bien conocida, solamente el arrecife Lobos cuenta con datos de la distribución bentónica y de la cobertura coralina (Rigby \& McIntire, 1966; Chávez et al. 1970; Chávez, 1973; Escobar-Vásquez \& Chávez, 2012), por lo tanto, es necesario generar información que permita conocer el estado de condición del resto de los arrecifes del SALT, dado que a pesar de que ya existe un plan de manejo (SEMARNAT \& CONANP, 2014), potencialmente están expuestos a perturbaciones naturales $\mathrm{y}$ 
humanas (Horta-Puga, 2010), por ejemplo, las actividades petroleras han modificado la estructura del arrecife Medio y Lobos, y el tránsito marítimo ha ocasionado daños, siendo el más reciente el encallamiento del barco "Mu Du Bong" en el arrecife Tuxpan (PROFEPA, 2014).

En este estudio se aportan los primeros datos de la cobertura del arrecife Enmedio, con el propósito de que sirvan de base para futuras decisiones en materia de manejo, conservación e investigación científica; cabe destacar que en dicho arrecife han ocurrido dos encallamientos de barcos en los últimos años (SEMARNAT \& CONANP, 2014) y presenta desarrollo de colonias de Acropora palmata y Acropora cervicornis (De la Cruz-Francisco et al. 2014), especies protegidas por la NOM-059-SEMARNAT, 2010.

\section{MATERIALES Y MÉTODOS}

Área de estudio: El arrecife Enmedio es de tipo de plataforma, se localiza a $14 \mathrm{~km}$ de la desembocadura del Río Tuxpan, es uno de los arrecifes más pequeños del SALT. Tiene una longitud de $1.06 \mathrm{~km}$ y su anchura es de $0.48 \mathrm{~m}$, en general presenta tres ambientes arrecifales (Fig. 1). La llanura arrecifal tiene una profundidad variable que alcanza hasta $2 \mathrm{~m}$; en este ambiente se desarrollan colonias de A. palmata, Porites furcata, Pseudodiploria clivosa y Milleropa alcicornis, también se destaca un parche de Eunicea flexuosa. El arrecife frontal presenta una pendiente suave y cubierta de roca coralina, mientras en el arrecife posterior dominan colonias de corales masivos, por ejemplo, Orbicella annularis, Colpophyllia natans y Siderastrea siderea (Tunnell, 2010; SEMARNAT \& CONANP, 2014).

Durante el periodo octubrediciembre del año 2014 y abril-julio del año 2015 se caracterizó la riqueza y cobertura bentónica del arrecife estableciendo cinco sitios de muestreo a lo largo de cada ambiente arrecifal y separados entre sí aproximadamente a $50 \mathrm{~m}$, debido a que el arrecife es pequeño. Como unidad de muestreo se utilizó un cuadrante de rejillas dividido en 100 cuadrados, donde cada cuadrado de $10 \mathrm{~cm}$ representó el $1 \%$ de cobertura relativa. A través de este instrumento se contabilizó el número de cuadrados ocupados por los siguientes grupos bentónicos: algas filamentosas, algas coralinas costrosas, macroalgas, esponjas, corales, zoántidos y octocorales, así como los componentes bentónicos: pavimento, escombros y arena, el primero se distingue por ser una superficie dura de coral muerto que potencialmente promueve el reclutamiento larval, mientras los dos últimos no son viables para el asentamiento larval dado que son fondos bentónicos no consolidados (Lang et al. 2010). Cabe aclarar que macroalgas, esponjas, corales, zoántidos y octocorales fueron estimados por especie debido a la facilidad de identificarlos en el campo por las características morfológicas y de coloración (Humann \& Deloach, 2002; Collin et al. 2005; Ocaña et al. 2007). Con base en estos criterios, los datos de riqueza y cobertura bentónica se recabaron colocando un 

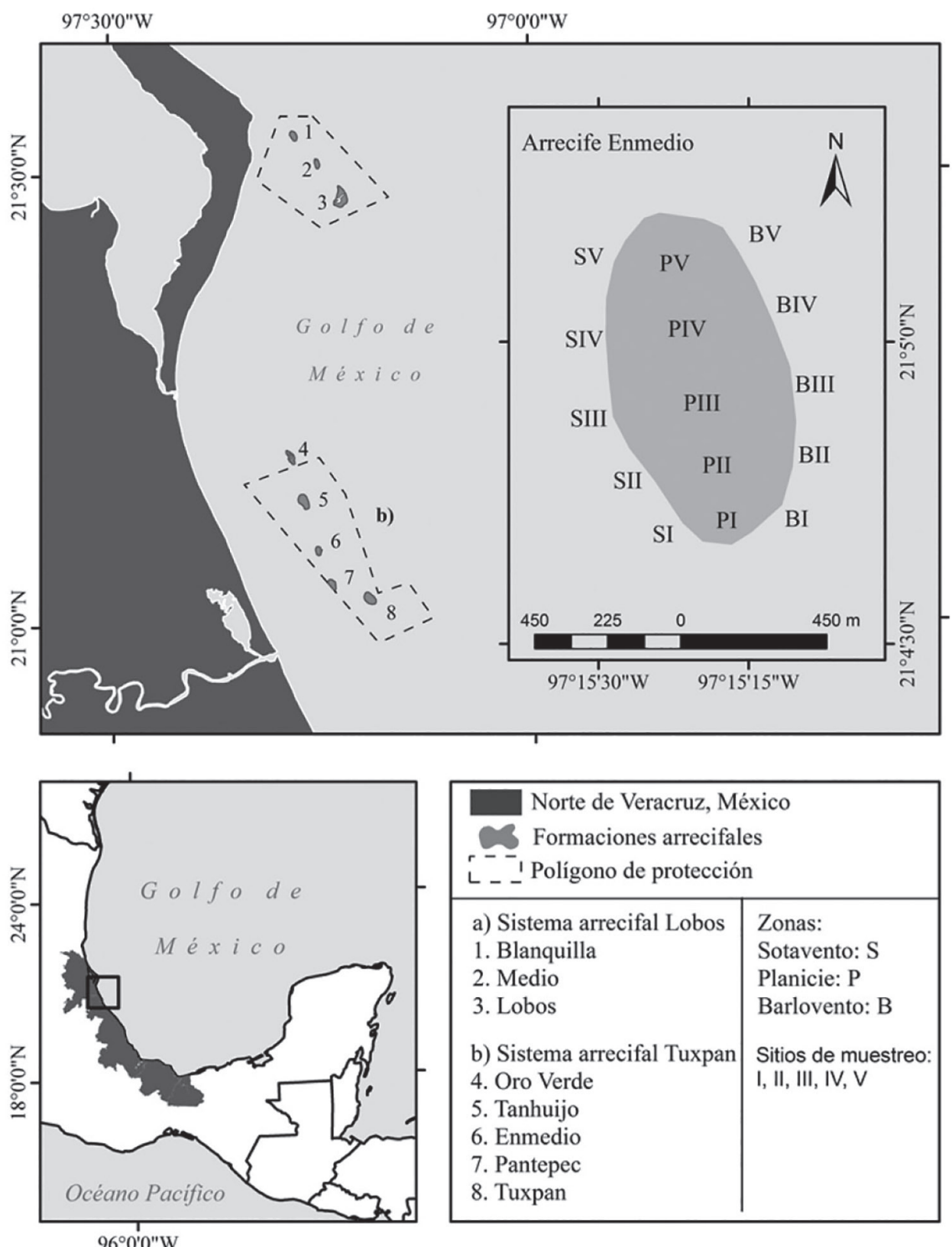

Norte de Veracruz, México

Formaciones arrecifales

L - I Polígono de protección

a) Sistema arrecifal Lobos Zonas:

1. Blanquilla

Sotavento: S

2. Medio

Planicie: P

3. Lobos

Barlovento: $\mathrm{B}$

b) Sistema arrecifal Tuxpan Sitios de muestreo:

4. Oro Verde

I, II, III, IV, V

5. Tanhuijo

6. Enmedio

7. Pantepec

8. Tuxpan

Fig. 1. Localización geográfica del arrecife Enmedio y distribución de los sitios de muestreo Fig. 1. Geographical location of Enmedio reef and distribution of sampling sites

cuadrante de $1 \mathrm{~m}^{2}$ a cada $5 \mathrm{~m}$ sobre tres transectos longitudinales de 50 $\mathrm{m}$, con el fin de obtener 33 muestras independientes, de tal manera que se estimó la cobertura de $33 \mathrm{~m}^{2}$ por sitio de muestreo. Tanto en el arrecife frontal como en el posterior los transectos fueron tendidos perpendicularmente, 
con la finalidad de abarcar un mayor intervalo de profundidad de 5-15 m, mientras en la planicie, al ser una área plana y amplia, los transectos se tendieron paralelos al arrecife.

Para determinar la eficiencia del muestreo en cada ambiente arrecifal, se calculó el porcentaje de representatividad del muestreo o también llamado índice de completitud, el cual consistió en dividir el valor de la riqueza observada (multiplicado por 100) entre el valor de la riqueza esperada del estimador no paramétrico Chao 1, asimismo, se consideró como eficiente el muestreo a partir de $90 \%$ de representatividad (Moreno \& Halffter, 2000); tanto los valores de la riqueza observada como de la estimada se obtuvieron con el programa EstimateS, versión 8 (Colwell, 2006) y se graficaron en un archivo Excel para representar la curva acumulativa de especies. También se evaluó el nivel de sesgo y exactitud del estimador con base en las fórmulas propuestas por Walther \& Moore (2005): sesgo= $\left(\mathrm{S}_{\text {estimada }}-\mathrm{S}_{\text {verdadera }}\right) / \mathrm{S}_{\text {verdadera }} ; \quad$ exactitud $=$ $\left(\left(\mathrm{S}_{\text {estimada }}-\mathrm{S}_{\text {verdadera }}\right) / \mathrm{S}_{\text {verdadera }}\right)^{2}$, en ambas fórmulas se obtienen valores entre -1 a 1 , cuando los valores son cercanos a cero indica menos sesgo o mayor exactitud, respectivamente.

Por otra parte, la cobertura relativa de los componentes bentónicos se promedió por ambiente arrecifal y por sitios expresándolos en valores porcentuales (\%). Asimismo, con la cobertura porcentual determinada por especie se estimaron los índices de riqueza específica, diversidad de Shannon, equitatividad y dominancia de Simpson, tanto de ambientes como de sitios de muestreo, utilizando el programa Primer V7 (Clarke \& Gorley, 2015); los valores obtenidos de la diversidad de Shannon se ocuparon para estimar la diversidad alfa verdadera de orden 1, la cual se obtiene a partir del exponente de Shannon, este índice expresa el número efectivo de especies que tendría una comunidad integrada por especies igualmente abundantes, dicho índice tiene la ventaja de realizar comparaciones de la diversidad verdadera de dos o más comunidades (Moreno et al. 2011).

Para determinar diferencias significativas entre los sitios de muestreo de cada ambiente arrecifal se realizaron análisis de varianza (ANOVA) con base en la cobertura (para los grupos mejor representados) y en los atributos ecológicos mencionados previamente utilizando el programa SPSS, versión 22.0 (IBM, 2013). Mediante estadística multivariada se realizó un análisis de similitud (ANOSIM) de la cobertura porcentual estimada por especie, con el objetivo de determinar diferencias significativas entre los ambientes arrecifales, los datos cuantitativos fueron transformados a raíz cuadrada para minimizar la varianza. Además, se utilizó el método de ordenación de escalamiento multidimensional no métrico (nMDS) con distancia de Bray-Curtis para representar gráficamente las similitudes de los grupos, asimismo, se realizó un SIMPER para detectar que especies contribuyen a la similitud de los grupos formados, todo el análisis multivariado se efectuó mediante el programa Primer V7 (Clarke \& Gorley, 2015). 
Cuadro 1. Cobertura de los componentes bióticos y abióticos del arrecife Enmedio Table 1. Coverage of biotic and abiotic substrates of Enmedio reef

\begin{tabular}{|c|c|c|c|c|c|}
\hline Componentes & $\%$ & Grupos & $\%$ & Grupos bentónicos & $\%$ \\
\hline \multirow[t]{8}{*}{ Bióticos } & 37.3 & Algas & 8 & Algas filamentosas & $3.9( \pm 4.1)$ \\
\hline & & & & $\begin{array}{l}\text { Algas coralinas } \\
\text { costrosas }\end{array}$ & $3.4( \pm 5)$ \\
\hline & & & & Macroalgas & $0.7( \pm 0.9)$ \\
\hline & & Esponjas & 0.5 & Esponjas & $0.5( \pm 0.5)$ \\
\hline & & Cnidarios sésiles & 28.8 & Corales & $19.8( \pm 18.8)$ \\
\hline & & & & Octocorales & $6.4( \pm 4.4)$ \\
\hline & & & & Anémonas & $0.2( \pm 0.2)$ \\
\hline & & & & Zoántidos & $2.4( \pm 4)$ \\
\hline \multirow{3}{*}{$\begin{array}{l}\text { Abióticos } \\
\text { (Sustrato } \\
\text { desnudo) }\end{array}$} & 62.7 & & 62.7 & Escombros & $7.4( \pm 4.1)$ \\
\hline & & & & Arena & $8.3( \pm 11)$ \\
\hline & & & & Pavimento & $47( \pm 23.4)$ \\
\hline Total & 100 & & 100 & & 100 \\
\hline
\end{tabular}

\section{RESULTADOS}

El bentos del arrecife Enmedio está representado por ocho componentes bióticos, los cuales acumulan un valor promedio de $37.3 \%$ de cobertura y lo representan 68 especies sésiles, siendo los corales los más representativos en cobertura y en número de especies. Sin embargo, se destaca la notable cobertura de los componentes abióticos $(62.7 \%)$ representados por escombros, arena y pavimento, siendo este último el más dominante en el arrecife (Cuadro 1). Esencialmente, las especies más importantes en cobertura fueron: O. annularis, Erytropodium caribaeorum, C. natans y P. clivosa, las cuales contribuyen con $20 \%$ de la cobertura total (Cuadro 2).

Los muestreos realizados en cada ambiente arrecifal se consideran aceptables, dado que el índice de completitud muestra valores superiores al $90 \%$ de representatividad, esto significa que faltaron pocas especies por registrar, tal como se aprecia en las curvas acumulativas de especies observadas y esperadas, donde el estimador Chao 1 mostró menor sesgo y mayor exactitud en determinar el número de especies esperadas. De esta manera, la mayor riqueza de especies se registró en el arrecife posterior con 48 especies y la menor fue en la planicie arrecifal con 26 (Fig. 2).

En relación con la cobertura bentónica por ambiente, en el arrecife posterior los corales son el componente biótico más importante con $39.2 \%$ $( \pm 19.7)$ de cobertura, sin embargo, manifestaron diferencias significativas entre los sitios (Cuadro 3), debido a que los sitios SIII y SIV con pendiente más profunda presentaron los valores más altos en cobertura coralina a diferencia del resto de los sitios que exhiben una 


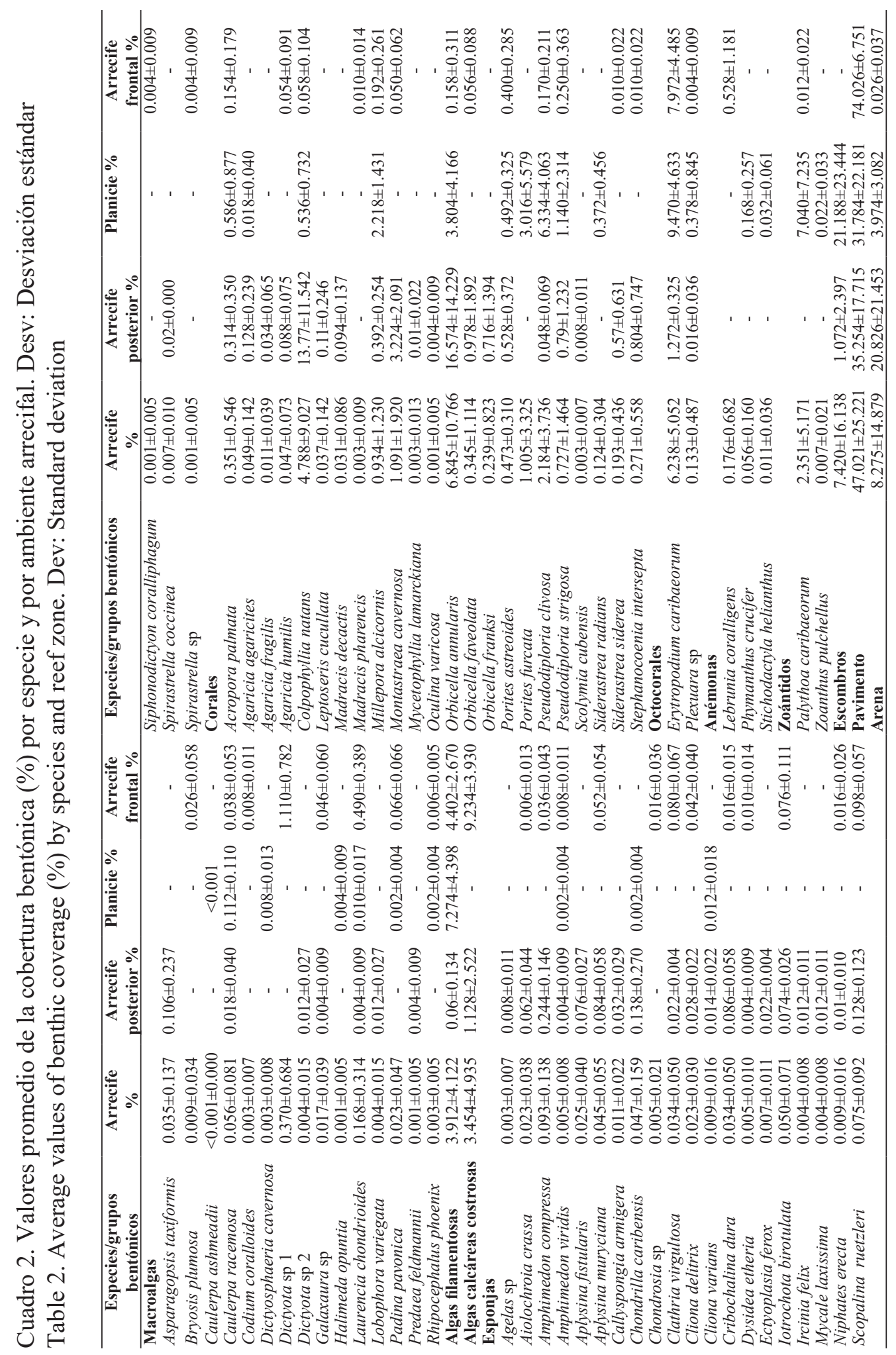




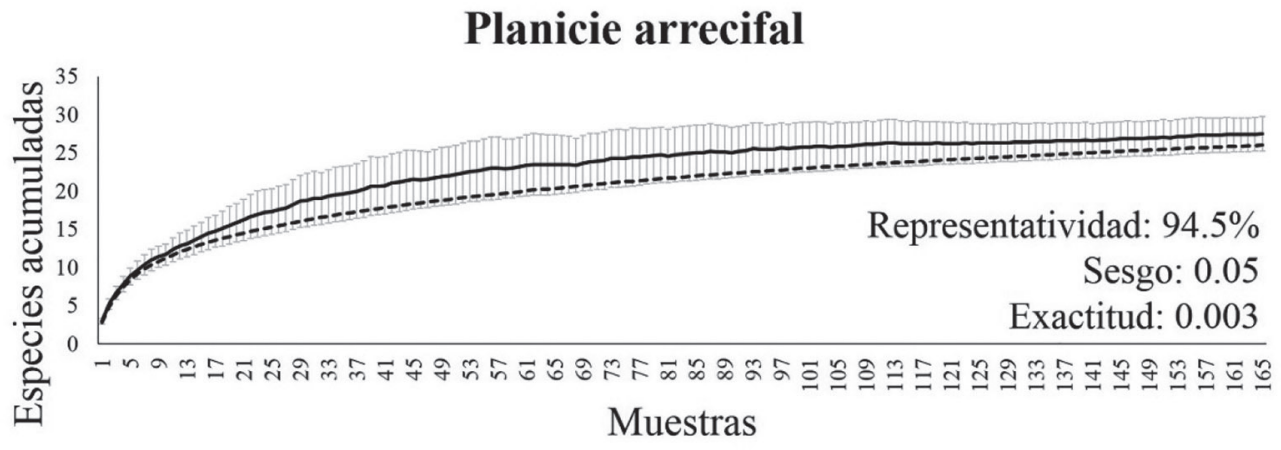

---Observadas -Chao 1

\section{Arrecife frontal}

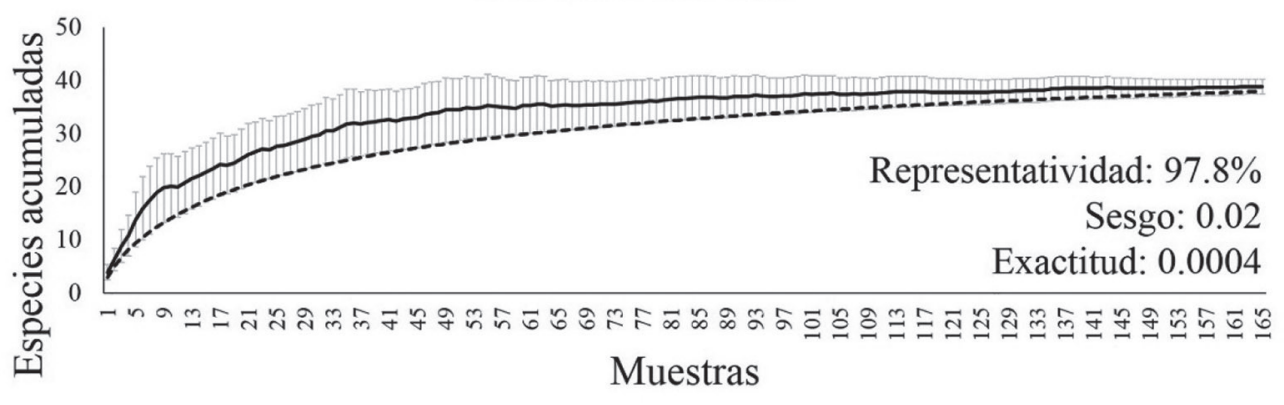

---Observadas -Chao 1

\section{Arrecife posterior}

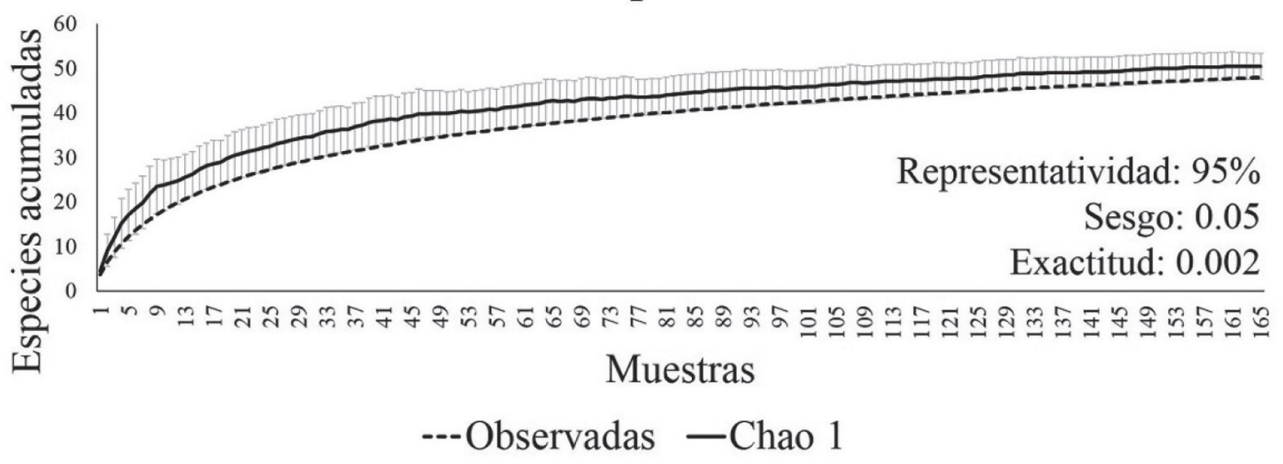

Fig. 2. Número de especies observadas y estimadas (Chao 1) para los tres ambientes del arrecife Enmedio, Sistema Arrecifal Lobos-Tuxpan. Las líneas punteadas negras indican el intervalo de confianza (superior e inferior) a $95 \%$ del estimador Chao 1

Fig. 2. Number of observed and estimated species (Chao 1) for the three environments at Enmedio reef, Lobos-Tuxpan Reef System. Black dotted lines indicate the confidence interval (upper and lower) to $95 \%$ of the Chao 1 estimator 
Cuadro 3. Valores de significancia de la prueba ANOVA correspondiente a la cobertura de los sitios de cada ambiente arrecifal

Table 3. Significance values of the ANOVA test corresponding to the coverage of the sites of each reef zone

\begin{tabular}{lllllll} 
& \multicolumn{2}{l}{ Arrecife posterior } & \multicolumn{2}{l}{ Planicie arrecifal } & \multicolumn{2}{c}{ Arrecife frontal } \\
\hline Componentes bióticos & $\mathrm{F}$ & Sig. & F & Sig. & F & Sig. \\
\hline Algas calcáreas costrosas & 1.989 & 0.172 & - & - & 2.044 & 0.164 \\
Algas filamentosas & 1.108 & 0.405 & 3.507 & 0.049 & 4.754 & 0.021 \\
Esponjas & 1.2 & 0.369 & 1 & 0.452 & 1.517 & 0.27 \\
Corales & 6.12 & 0.009 & 0.95 & 0.475 & 2.374 & 0.122 \\
Octocorales & 0.188 & 0.939 & 5.448 & 0.014 & 1.52 & 0.269 \\
\hline
\end{tabular}

corta pendiente y están caracterizados por la dominancia de arena y pavimento (Fig. 2). Las especies que caracterizan esta pendiente arrecifal son: $O$. annularis, $C$. natans y $M$. cavernosa, las cuales contribuyen con el 33.6\% de la cobertura (Cuadro 2).

En cambio en el arrecife frontal, la cobertura biótica se reduce en promedio a $26 \%$, y lo constituyen esencialmente algas coralinas costrosas, octocorales y algas filamentosas, el resto lo compone pavimento con 74\% $( \pm 6.7)$ (Cuadro 2); los sitios BIV y BV son los que manifestaron mayor cobertura biótica (Fig. 3), siendo las algas filamentosas el único grupo que presentó diferencias significativas entre los sitios (Cuadro 3). E. caribaeorum es la especie más importante en cobertura para este ambiente arrecifal (Cuadro 2).

Mientras tanto, la planicie arrecifal presentó una cobertura biótica promedio de $43 \%$, y lo constituyen en orden de importancia: corales, octocorales, algas filamentosas y zoántidos, de los cuales solamente manifestaron diferencias significativas los octocorales y macroalgas por la baja cobertura re- gistrada en los sitios BI y BV, respectivamente (Cuadro 3). Es importante destacar que estos grupos bentónicos definen una notable zonación en la planicie representadas sobre todo por especies de cnidarios, de tal manera que al sur de la planicie (PI) la cobertura está representada principalmente por $E$. caribaeorum $(9.9 \%)$, P. clivosa $(8.9 \%)$ y $P$. strigosa $(5.3 \%)$, adentrándose a la llanura arrecifal (SII) predomina $E$. caribaeorum (12.3\%) y O. annularis $(10.9 \%)$, en la parte central de la planicie (PIII) se delimita otra zonación compuesta por $P$. porites $(13 \%)$ y $P$. clivosa $(11.9 \%)$, en este lugar también se destaca la cobertura de escombros que se extiende al sitio IV, donde E. caribaeorum y $P$. caribaeorum caracterizan dicha área y amplían su cobertura hacia el extremo norte de la planicie donde la prevalencia de pavimento es notable en el sitio V (Fig. 3).

En cuanto a los atributos ecológicos, la riqueza promedio no manifestó diferencias significativas en la planicie arrecifal $(\mathrm{F}=0.733 ; P=$ $0.590)$, en el arrecife posterior $(\mathrm{F}=$ 2.395; $P=0.120)$ y en el frontal $(\mathrm{F}=$ 


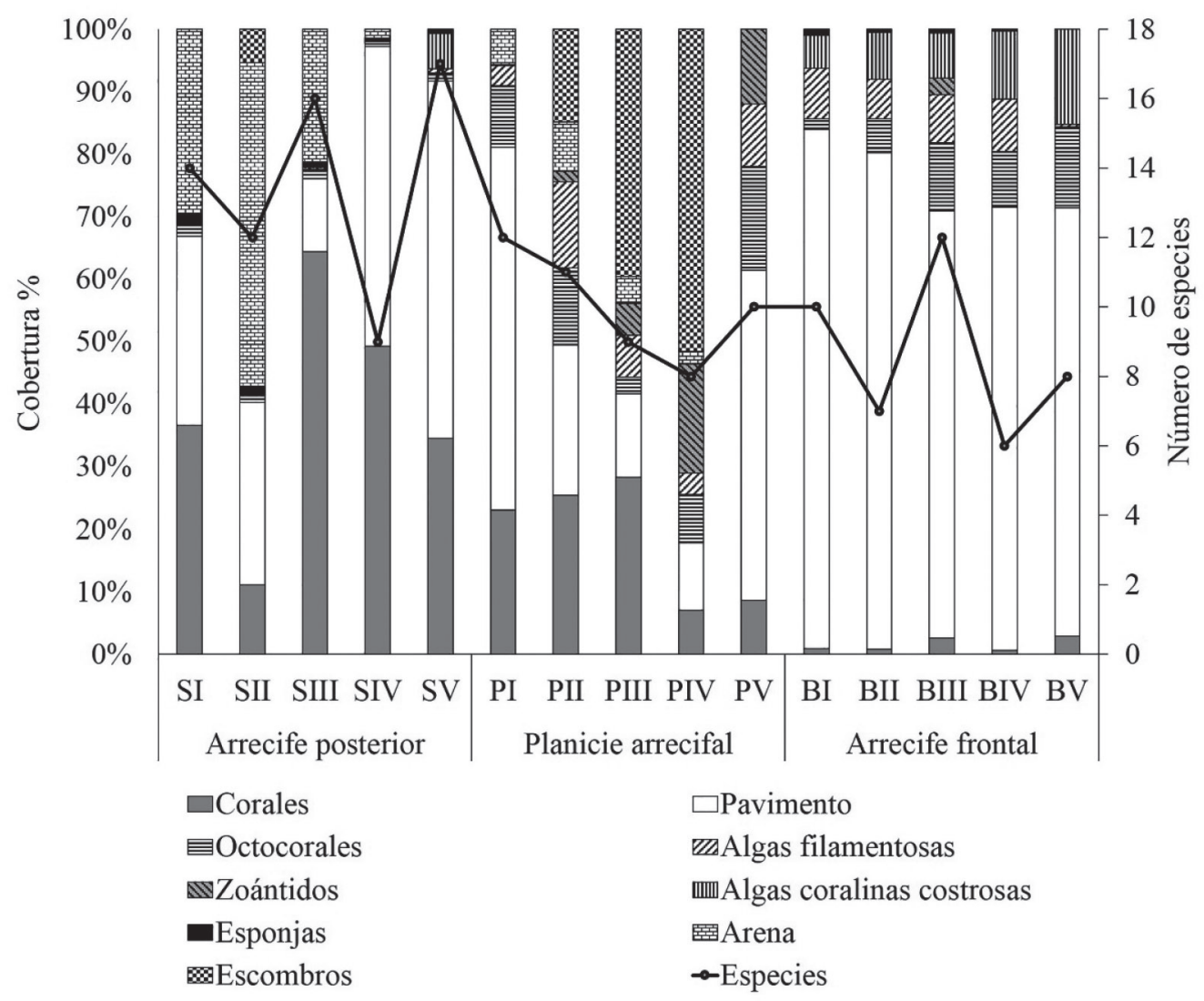

Fig. 3. Valores de riqueza y cobertura de los sustratos bióticos y abióticos por sitios de muestreo, en el arrecife Enmedio. Sitios de muestreo: I, II, III, IV y V

Fig. 3. Richness and coverage values of biotic and abiotic substrates by sampling sites on Enmedio reef. Sampling sites: I, II, III, IV and V

2.426; $P=0.117)$. En relación con la equitatividad, dominancia de Simpson y diversidad verdadera, en el arrecife posterior los sitios SI, SII, SIII y SV mostraron tendencia a la equitatividad y presentaron mayor número de especies efectivas, a diferencia del sitio SIV que manifestó mayor valor de dominancia y menor diversidad verdadera (Fig. 4); estos contrastes son estadísticamente significativos para la equitatividad $(\mathrm{F}=4.149 ; P=0.03)$, pero no para la dominancia $(\mathrm{F}=1.253 ; P=0.350)$.
En el arrecife frontal, únicamente el sitio BI manifestó equitatividad y mayor número de especies efectivas, dado que la comunidad bentónica de los sitios BII y BV resultaron ser dominantes y menos diversos en especies efectivas, esencialmente el sitio BIV registró mayor dominancia $\mathrm{y}$, por consiguiente, menor diversidad verdadera (Fig. 4), sin embargo, tanto la equitatividad como la dominancia no manifestaron diferencias significativas entre los sitios $(\mathrm{F}=1.545, P=0.262$; 


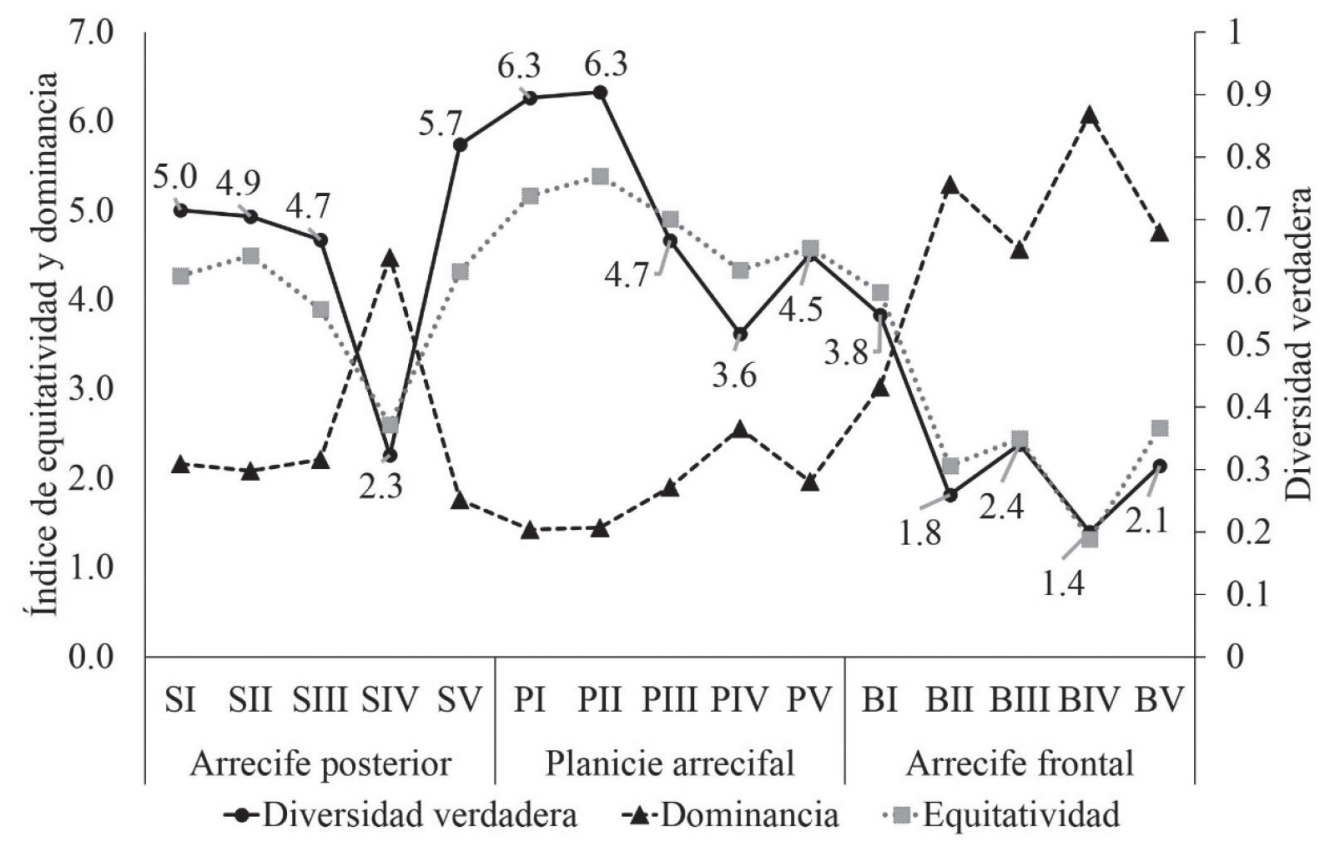

Fig. 4. Valores de dominancia, equitatividad y diversidad verdadera por sitios de cada ambiente del arrecife Enmedio. Sitios de muestreo: I, II, III, IV y V

Fig. 4. Values of dominance, evenness and true diversity by site at each environment of Enmedio reef. Sampling sites: I, II, III, IV and V

$\mathrm{F}=0.971, P=0.465$, respectivamente). Mientras tanto, en la planicie arrecifal la dominancia es baja en los sitios, por lo tanto, la equitatividad y la diversidad verdadera son notables al extremo sur (PI y PII) y tienden a decrecer ligeramente en los sitios PIII y PV caracterizados por la prevalencia de arena, escombros y pavimento (Fig. 4). Estadísticamente, la dominancia y la equitatividad tampoco mostraron diferencias significativas $(\mathrm{F}=1.364$, $P=0.313 ; \mathrm{F}=3.420, P=0.052$ ).

Por otra parte, el análisis ANOSIM determina la existencia de diferencias significativas entre sotavento, planicie y barlovento (R global $=0.961, P<0.0001$ ) con base en la cobertura bentónica estimada por especies. El método de ordenación con distancia de Bray-Curtis muestra claramente la separación de los ambientes arrecifales (Fig. 5). Siendo sotavento el grupo más disímil junto con barlovento $(65.49 \%)$ y planicie (66.57\%). Con base en el análisis SIMPER, cuatro especies contribuyen a la similitud de la planicie (79.47\%), cuatro a sotavento $(72.36 \%)$ y dos a barlovento (73.23\%) (Cuadro 4).

\section{DISCUSIÓN}

Los corales constituyen el componente biótico más importante para el arrecife Enmedio dado que manifestaron mayor cobertura bentónica con $19.8 \% \quad( \pm 18.8)$ dicha cifra se considerarelevante debidoa que envarias 
Cuadro 4. Valores de similitud porcentual (SIMPER) de las especies que contribuyen a la semejanza en las zonas arrecifales con base en la distancia de Bray-Curtis Table 4. Similarity percentages (SIMPER) of the species that contribute to similarity in the reef zones based on Bray-Curtis' distance

\begin{tabular}{lllll}
\hline Zonas & Especies & Similaridad promedio & \% Contribución & \% Acumulado \\
\hline Planicie & E. caribaeorum & $16.56 \pm 3.39$ & 26.40 & 26.40 \\
$(79.47 \%)$ & P. clivosa & $12.31 \pm 3.82$ & 19.63 & 46.03 \\
& P. caribaeorum & $8.62 \pm 1.15$ & 13.74 & 59.77 \\
& O. annularis & $7.58 \pm 2.29$ & 12.08 & 71.85 \\
\hline Arrecife & O. annularis & $18.85 \pm 5.26$ & 28.49 & 28.49 \\
posterior & C. natans & $17.50 \pm 3.21$ & 26.45 & 54.93 \\
$(72.36 \%)$ & M. cavernosa & $7.33 \pm 2.07$ & 11.07 & 66.00 \\
& E. caribaeorum & $5.71 \pm 6.39$ & 8.64 & 7464 \\
\hline Arrecife frontal & E. caribaeorum & $45.06 \pm 6.05$ & 66.28 & 66.28 \\
$(73.23 \%)$ & P. astreoides & $8.87 \pm 4.62$ & 13.04 & 79.33 \\
\hline
\end{tabular}

formaciones arrecifales de Veracruz se han reportado valores inferiores como en el arrecife Hornos con $6.5 \%$, Cabezo con $11.1 \%$ y Chopas con $16.8 \%$, no obstante, la cobertura coralina que presenta el arrecife Enmedio es parecida al promedio general que se reporta para el SAV con $18.37 \%-19.1 \%$ (datos de Pérez-España et al. 2015 y Horta-Puga et al. 2015, respectivamente) y para el Atlántico Occidental con 20.5\% (HortaPuga \& Tello-Musi, 2009; HortaPuga et al. 2015). A excepción de los arrecifes Flower Garden Banks que han mantenido la cobertura por encima del 50\% (Jackson et al. 2014; Johnston et al. 2014), los arrecifes del Golfo de México y varios arrecifes del Caribe aún manifiestan bajos valores en cobertura coralina, y se atribuyen a la frecuencia de perturbaciones naturales y de origen humano (Jackson et al. 2014).

Se destaca mucho la sedimentación a la que están sometidos los arrecifes de Veracruz (Horta-Puga \& Tello-
Musi, 2009) dado que es un elemento que propicia el enriquecimiento de nutrientes y, con ello, la proliferación de algas (McClanahan et al. 2002), sin embargo, las algas filamentosas y algas calcáreas costrosas presentaron baja cobertura (8\%) en el arrecife Enmedio; esto podría deberse a que las formaciones coralinas del sistema arrecifal Tuxpan son oligotróficas y con baja productividad primaria (SalasPérez et al. 2015), no obstante, la proliferación de algas en los arrecifes de Veracruz ha sido citada en el arrecife Lobos con 44.7\% (Escobar-Vásquez \& Chávez, 2012) y en los arrecifes del SAV presentando coberturas superiores a 50\% (Jones et al. 2008).

Si bien la cobertura coralina es parecida a la del SAV, en cuanto a las especies de corales dominantes difieren un poco, dado que en el arrecife Enmedio las especies O. annularis, $C$. natans y $P$. clivosa manifestaron mayor cobertura, en cambio para el 


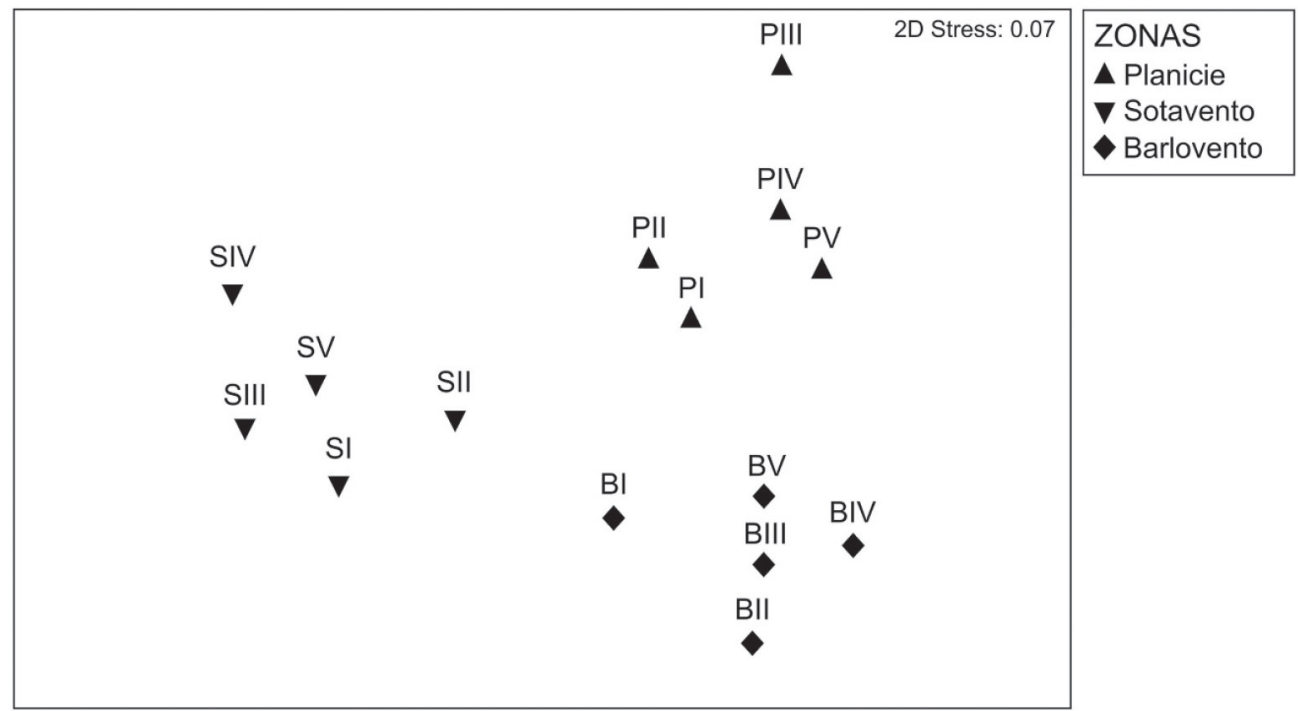

Fig. 5. Análisis de escalamiento multidimensional no métrico (nMDS) aplicado a los sitios (I, II, III, IV y V) de las zonas arrecifales: sotavento (S), planicie (P) y barlovento (B) Fig. 5. Analysis of non-metric multidimensional scaling (nMDS) applied to the sites (I, II, III, IV and V) in the reef areas: Leeward (S), Reef flat (P) and Windward (B)

SAV se reconocen como dominantes a C. natans, Montastraea cavernosa y O. faveolata (Horta-Puga et al. 2015; Pérez-España et al. 2015).

Las discrepancias que ocurren en los ambientes del arrecife Enmedio con base en la riqueza y cobertura bentónica, también han sido reportadas fundamentalmente para los arrecifes de Anegada, Isla de Enmedio y Cabezo (Horta-Puga et al. 2015); estos contrastes en los ambientes arrecifales son normales dado que coinciden con la zonación típica descrita para los arrecifes del Golfo de México y Mar Caribe por Jordán-Dahlgren (1993) y Chávez et al. (2010), no obstante, algunos arrecifes presentan ciertas excepciones (Pérez-España et al. 2015).

El arrecife posterior es el ambiente arrecifal más importante por concentrar la mayor riqueza de especies y el máximo desarrollo coralino (39.2\% \pm 19.7 ); este mismo patrón biológico se ha reportado para los arrecifes del SAV con $32.7 \%( \pm 13.0)$, siendo claros ejemplos los arrecifes de Cabezo, Anegada, Isla de Enmedio, Isla Verde y Gallega (Horta-Puga et al. 2015). Las especies que manifestaron mayor cobertura coralina fueron: $O$. annularis, C. natans y M. cavernosa; este conjunto de especies son típicas del citado ambiente arrecifal y son considerados como los principales constructores de arrecifes después de los acropóridos (Escobar-Vásquez \& Chávez, 2012; Horta-Puga et al. 2015), debido a que son especies que resisten la sedimentación y escasez de luz, por 
presentar coralitos grandes y colonias en forma de domo (Darling et al. 2012). Se argumenta que el oleaje moderado, la pendiente más pronunciada y la constante sedimentación condicionan esta zonación arrecifal (Chávez et al. 2010). Asimismo, el conjunto de especies que caracterizan el arrecife posterior determinan un ambiente diverso en número de especies efectivas y con tendencia a la equitatividad, dicha condición ecológica también se ha reportado para los arrecifes del $\mathrm{SAV}$, pero con valores importantes de equitatividad $\left(\mathrm{J}^{\prime}=0.74\right)$ de acuerdo con Horta-Puga et al. (2015).

Mientras tanto, la condición bentónica que manifiesta el arrecife frontal en relación con la baja riqueza y cobertura coralina, también ha sido reportada para varios arrecifes del SAV, como en los arrecifes de Anegada, Cabezo, Isla de Enmedio y Gallega (Horta-Puga et al. 2015). Este patrón biológico es el resultado de cambios ocurridos en décadas pasadas, fundamentalmente por la mortandad masiva de A. palmata (Jordán-Dahlgren, 1993; Jordán-Dahlgren \& RodríguezMartínez, 2003), especie que dominaba este ambiente arrecifal en todo el Atlántico y que hasta la fecha no ha mostrado signos de recuperación; por consiguiente, ahora esta zona muerta es recubierta por organismos de rápido crecimiento, como algas filamentosas, algas coralinas costrosas y octocorales incrustantes, los cuales cumplen la función de cimentar y estabilizar el sustrato (Sebens \& Miles, 1988; JordánDahlgren \& Rodríguez-Martínez,
2003; Silva et al. 2015), justamente estos tres componentes bentónicos caracterizan la pendiente frontal del arrecife Enmedio, pero condicionan un entorno dominante y con valores bajos de diversidad verdadera en todos los sitios de muestreo. Esta condición arrecifal es contraria a la que se reporta para el SAV, dado que la equitatividad es alta en este sistema arrecifal con un valor promedio de $\mathrm{J}^{\prime}=0.82$ (HortaPuga et al. 2015).

La planicie arrecifal también es importante en cobertura por componentes bióticos después del arrecife posterior, sin embargo, se reparte en varios grupos bentónicos, donde los corales resultan ser los de mayor cobertura con $18.5 \%$ ( \pm 9.9$)$; esta cifra es superior al promedio general que se reporta para el SAV con $9.4 \%( \pm 8.2)$, que incluso en varios arrecifes de este sistema arrecifal cuentan con reportes más bajos: Blanca $(7.1 \% \pm 5.5)$, Cabezo $(2.2 \% \pm 2.4)$, Chopas $(4.7 \% \pm 2.3)$, Isla Enmedio $(2.8 \% \pm 2.3)$, Isla Sacrificios $(4.1 \% \pm 3.2)$, Pájaros $(11.2 \pm 6.5)$, sin embargo, Isla Verde es el único arrecife que se destaca con 30.3\% $( \pm 13.2)$ de acuerdo con datos obtenidos por HortaPuga et al. (2015).

Específicamente, la prevalencia de corales ocurre en los sitios I, II y III, lo cual se atribuye a la presencia de las especies $P$. clivosa, $P$. furcata, $O$. annularis y $M$. alcicornis, que son típicas de las áreas someras dado que tienen adaptaciones para abatir el oleaje, como la plasticidad morfológica y la presencia de simbiontes para tolerar la intensidad de la luz (Kaandorp, 1999; LaJeunesse, 
2002; Darling et al. 2012), factores que ocurren en este ambiente arrecifal (Jordán-Dahlgren, 1993). En cambio en los sitios IV y V ocurre lo contrario, se destacan la cobertura de escombros y el pavimento coralino, respectivamente, y solo especies incrustantes, competidoras por el espacio y de rápido crecimiento, como E. caribaeorum y $P$. caribaeorum (Suchanek \& Green, 1981; Sebens \& Miles, 1988; Silva et al. 2015), caracterizan esta parte del arrecife. Cabe mencionar que solamente $P$. clivosa y $P$. furcata han sido citadas como dominante y subdominante, respectivamente, para la planicie de los arrecifes Lobos y Tuxpan por Jordán-Dahlgren \& RodríguezMartínez (2003).

El conjunto de especies que caracterizan la planicie del arrecife Enmedio condicionan una comunidad equitativa $\mathrm{y}$, por tanto, diversa en número de especies efectivas; este patrón ecológico también se ha reportado para varios arrecifes del SAV como Blanca, Hornos y Gallega, sin embargo, existen algunas excepciones dado que los arrecifes Isla de Enmedio, Isla Sacrificios y Chopas presentan reportes bajos de equitatividad (Horta-Puga et al. 2015); esto sugiere que en dichos arrecifes coralinos se establecen especies dominantes en la planicie arrecifal.

Por otra parte, el análisis de ordenación (nMDS) y el ANOSIM reafirman que los ambientes arrecifales son estadísticamente diferentes y se atribuyen a las disparidades en riqueza y cobertura bentónica que manifestó cada área arrecifal. Las especies que causan la similitud de los sitios de sotavento son precisamente las de mayor cobertura compuestas por corales masivos $(O$. annularis, $C$. natans y $M$. cavernosa) de crecimiento lento, pero adaptadas al estrés por la sedimentación y baja luminosidad (LaJeunesse, 2002; Darling et al. 2012), mientras en la planicie la similitud de los sitios la causan especies (E. caribaeorum, $P$. clivosa, O. annularis y P. caribaeorum) que son de rápido crecimiento y que toleran el oleaje y la alta luminosidad adquiriendo formas principalmente incrustantes y aplanadas (Suchanek \& Green, 1981; Sebens \& Miles, 1988; Kaandorp, 1999; La-Jeunesse, 2002; Darling et al. 2012; Silva et al. 2015), en cambio la agrupación de los sitios de barlovento la origina otra comunidad compuesta por octocorales incrustantes (E. caribaeorum) y corales aplanados ( $P$. astreoides).

Las comunidades bentónicas que se distinguen en los ambientes del arrecife Enmedio (sotavento, planicie y barlovento) son parecidas a la zonación que se describe para el arrecife Lobos y el SAV (Chávez et al. 2010; Horta-Puga et al. 2015), resultando importantes el arrecife posterior y la planicie arrecifal en diversidad de especies y en cobertura bentónica, que al parecer están determinadas por la influencia del oleaje, la profundidad, la luz y las características del sustrato inerte, todos estos atributos sitúan al arrecife Enmedio como un ecosistema importante para el Sistema Arrecifal Lobos-Tuxpan. 


\section{AGRADECIMIENTOS}

Agradecemos a Blanca Esther Raya Cruz y Arturo Serrano Solís por facilitarnos el equipo de buceo y la embarcación de la Facultad de Biología, así como también a Liliana Flores Galicia por su apoyo en el resumen en inglés. Al personal que labora en el Club Náutico Tampamachoco y en el Club Náutico Aqua Sport por la amabilidad $\mathrm{y}$ el apoyo que siempre mostraron al recibirnos y atendernos en todo momento. A los revisores anónimos por sus comentarios y sugerencias.

\section{BIBLIOGRAFÍA}

Beltrán-Torres, A. \& Carricart-Ganivet, J. P. (1999). Lista revisada y clave para los corales pétreos zooxantelados (Hydrozoa: Milleporina; Anthozoa: Scleractinia) del Atlántico mexicano. Rev. Biol. Trop., 47(4), 813-829.

Burke, L., Reytar, K., Spalding, M. \& Perry, A. (2011). Reefs at Risk. Washington, D. C., EE. UU.: World Resources Institute.

Chamberlain, C. (1966). Some Octocorallia of Isla Lobos, Veracruz, Mexico. Brigham Young Univ. Geol. Stud., 13, 47-54.

Chávez, E. A. (1973). Observaciones generales sobre las comunidades del arrecife Lobos, Veracruz. An. Esc. Nac. Cien. Biol., 20, 13- 21.

Chávez, E. A., Hidalgo, E. \& Sevilla, M. L. (1970). Datos acerca de las comunidades bentónicas del arrecife Lobos, Veracruz. Rev. Soc. Mex. Hist. Nat., 30, 211-281.

Chávez, E. A., Tunnell, J. W. Jr. \& Withers, K. (2010). Zonación y Ecología de los Arrecifes: Plataforma Veracruzana y Banco Campeche. En J. W. Tunnell Jr., E. A. Chávez \& K. Withers (Eds.), Arrecifes Coralinos del Sur del Golfo de México (pp. 60-100). México: Instituto Politécnico Nacional.

Clarke, K. R. \& Gorley, R. N. (2015). Getting started with PRIMER V7. PRIMER-E. Plymouth, Reino Unido. PRIMER-E Ltd.

Collin, R., Díaz, M. C., Nuremburg, J., Rocha, M. R., Sánchez, J. A., Schulze, A., Schwartz, M. \& Valdez, A. (2005). Photographic Identification Guide to Some Common Marine Invertebrates of Bocas Del Toro, Panama. Caribb. J. Sci., 41(3), 638-707.

Colwell, R. K. (2006). EstimateS: Statistical estimation of species richness and shared species from samples. Versión 8.2. Recuperado en enero 12, 2016, disponible en http://viceroy.eeb.uconn. edu/estimates/EstimateSPages/EstimateSRegistration.htm

Darling, E. S., Alvarez-Filip, L., Oliver, T. A., McClanahan, T. R. \& Coté, I. M. (2012). Evaluating life-history strategies of reef corals from species traits. Ecol.Lett., 15, 1378-1386.http://dx.doi. org/10.1111/j.1461-0248.2012.01861.x De la Cruz-Francisco, V., Argüelles-Jiménez, J., González-Gándara, C. \& Pérez-España, H. (2014). Invertebrados: Acropora cervicornis y Acropora palmata. En F. Hernández-Baz \& D. U. Rodríguez-Vargas (Eds.), Libro Rojo de la Fauna del Estado de Veracruz (pp. 104-107). Xalapa, México: Gobierno del Estado de Veracruz, Procuraduría Estatal de Protección al Medio Ambiente, Universidad Veracruzana.

Escobar-Vásquez, C. \& Chávez, E. (2012, july). Coral community structure at Isla Lobos reef, Gulf of Mexico. Proceedings of the 12th International Coral Reef Symposium, Cairns, Australia.

González-Gándara, C., Cruz-Arellano, M., Domínguez-Barradas, C., Serrano, 
A. \& Basáñez-Muñoz, A. J. (2007). Macroalgas asociadas a cuatro hábitats del arrecife Tuxpan, Veracruz, México. Rev. Cient. UDO Agrí., 7(1), 252-257.

González-Gándara, C., Domínguez-Barradas, C., De la Cruz-Francisco, V., Solís-Marín, F. A. \& Carricart-Ganivet, J. P. (2015). Esponjas, corales escleractinios, equinodermos y peces de arrecifes coralinos del norte y sur de Veracruz. México: SNIB-CONABIO.

González-Gándara, C., Patiño-García, A., Asís-Anastacio, U., Serrano, A. \& Gómez, P. (2009). Lista de esponjas marinas asociadas al arrecife Tuxpan, Veracruz, México. Rev. Mex. Biod., 80, 1-5. Horta-Puga, G. \& Carricart-Ganivet, J. P. (1993). Corales pétreos recientes (Milleporina, Stylasterina y Scleractinia) de México. En S. I. Salazar-Vallejo \& N. E. González (Eds.), Biodiversidad Marina y Costera de México (pp. 6680). México: Com. Nal. Biodiversidad y CIQRO.

Horta-Puga, G. \& Tello-Musi, J. L. (2009). Sistema Arrecifal Veracruzano: condición actual y programa permanente de monitoreo: Primera etapa. México: Universidad Nacional Autónoma de México.

Horta-Puga, G. (2010). Impactos ambientales. En J. W. Jr. Tunnell, E. A. Chávez \& K. Withers (Eds.), Arrecifes Coralinos del Sur del Golfo de México (pp. 183-205). México: Instituto Politécnico Nacional.

Horta-Puga, G., Tello-Musi, J. L., Beltrán-Torres, A., Carricart-Ganivet, J. P., Carriquiry, J. D. \& VillaescusaCelaya, J. (2015). Veracruz Reef System: a hermatypic coral community thriving in a sedimentary terrigenous environment. En A. Granados-Barba, L. Ortiz-Lozano, D. Salas-Monreal \&
C. González-Gándara (Eds.), Aportes al conocimiento del Sistema Arrecifal Veracruzano: hacia el Corredor Arrecifal del Suroeste del Golfo de México (pp. 181-208). México: Universidad Autónoma de Campeche.

Humann, P. \& Deloach, N. (2002). Reef Creature Identification Florida Caribbean Bahamas (2nd. ed.). Florida, EE. UU.: New World Publications. Jack Sonville.

IBM Corp. (2013). IBM SPSS Statistics for Windows, Version 22.0. Armonk, New York, EE. UU.: IBM Corp.

Jackson, J. B. C., Donovan, M. K., Cramer, K. L. \& Lam, W. (2014). Status and Trends of Caribbean Coral Reefs: 1970-2012. Switzerland: Global Coral Reef Monitoring Network, IUCN, Gland.

Johnston, M. A., Nuttall, M. F., Eckert, R. J. \& Embesi, J. A. (2014). LongTerm Monitoring at East and West Flower Garden Banks National Marine Sanctuary: 2013 Annual Report. U.S. Galveston, EE. UU.: Department of Commerce, National Oceanic and Atmospheric Administration, Flower Garden Banks National Marine Sanctuary.

Jones, J., Withers, K. \& Tunnell, J. W. Jr. (2008, julio). Comparison of Benthic Communities on Six Coral Reefs in the Veracruz Reef System (Mexico). Proceedings of the 11th International Coral Reef Symposium, Ft., Lauderdale, Florida, $18,757-760$.

Jordán-Dahlgren, E. \& Rodríguez-Martínez, R. E. (2003). The Atlantic Coral Reefs of Mexico. En J. Cortés (Ed.), Latin American Coral Reefs (pp. 131-158). Amsterdam: Elsevier Science. http://dx.doi.org/10.1016/ B978-044451388-5/50007-2 
Jordán-Dahlgren, E. (1993). El ecosistema arrecifal coralino del Atlántico Mexicano. Rev. Soc. Mex. Hist. Nat., 44, 157-175.

Jordán-Dahlgren, E. (2002). Gorgonian distribution patterns in coral reef environments of the Gulf of Mexico: evidence of sporadic ecological connectivity? Coral Reefs, 21, 205-215.

Kaandorp, J. A. (1999). Morphological analysis of growth forms of branching marine sessile organisms along environmental gradients. Mar. Biol., 134, 295-306. http://dx.doi.org/10.1007/ s002270050547

LaJeunesse, T. C. (2002). Diversity and community structure of symbiotic dinoflagellates from $\mathrm{Ca}-$ ribbean coral reefs. Mar. Biol., 141, 387-400. http://dx.doi.org/10.1007/ s00227-002-0829-2

Lang, J. C., Marks, K. W., Kramer, P. A., Kramer, P. R. \& Ginsburg, R. N. (2010). AGGRA protocols version 5.4. Miami, EE. UU.: Atlantic and Gulf Rapid Reef Assessment.

Lewis, J. B. (1989). The ecology of Millepora. Coral Reefs, 8, 99-107. http:// dx.doi.org/10.1007/BF00338264

McClanahan, T. R., Cokos, B. A. \& Sala, E. (2002). Algal growth and species composition under experimental control of herbivory, phosphorus and coral abundance in Glovers Reef, Belize. Mar. Poll. Bull., 44, 441-451. http://dx.doi. org/10.1016/S0025-326X(02)00051-6

Moreno, C. \& Halffter, G. (2000). Assessing the completeness of bat biodiversity inventories using species accumulation curves. J. Applied Ecol., 37, 149-158. http://dx.doi.org/10.1046/ j.1365-2664.2000.00483.x

Moreno, C. E., Barragán, F., Pineda, E. \& Pavón, N. P. (2011). Reanálisis de la diversidad alfa: alternativas para interpretar y comparar información sobre comunidades ecológicas. Rev. Mex. Biodiv., 82, 1249-1261.

Ocaña, O., Moro, L., Ortega, J., Espinosa, J. \& Caballer, M. (2007). Guía visual de la biodiversidad marina de Guanahacabibes. I. Anémonas (Anthozoa: Actiniaria, Corallimorpharia, Ceriantharia y Zoanthidae). Avicennia, 19, 133-142.

Ortiz-Lozano, L., Pérez-España, H., Granados-Barba, A., González-Gándara, C., Gutiérrez-Velázquez, A. \& Martos, J. (2013). The Reef Corridor of the Shouthwest Gulf of Mexico: Challenges for its management and conservation. Ocean. Coast. Manage., 86, 22-32. http://dx.doi.org/10.1016/j. ocecoaman.2013.10.006

Pérez-España, H., Ávila-Gutiérrez, P. S., Melo-Merino, S. M., Berumen-Solórzano, P. \& Flores-Arévalo, R. R. (2015). Patrones interanuales e interarrecifales de las comunidades de peces, corales y equinodermos en el Sistema Arrecifal Veracruzano. En A. Granados-Barba, L. Ortiz-Lozano, D. Salas-Monreal \& C. González-Gándara (Eds.), Aportes al conocimiento del Sistema Arrecifal Veracruzano: hacia el Corredor Arrecifal del Suroeste del Golfo de México (pp. 157-178). México: Universidad Autónoma de Campeche.

PROFEPA. (2014). Daño Mu Du Bong superficie de 3,662 M2 Y 250 colonias de especies en ANP "LOBOS-TUXPAN": PROFEPA. Recuperado en diciembre 18, 2015, en http://www.profepa.gob.mx/innovaportal/v/6179/1/ $\mathrm{mx} /$ dano_mu_du_bong_superficie_ de_3662_m2_y_250_colonias_de_especies_en_anp_\%E2\%80\%9 tuxpan $\% \mathrm{E} 2 \% 80 \% 9 \mathrm{D}$ : profepa.html 
Quintana, M. J. (1991). Resultados del programa de investigaciones en arrecifes veracruzanos del Laboratorio de Sistemas Bentónicos Litorales. Hidrobiológica, 1(1), 73-86.

Rigby, J. K. \& McIntire, W. G. (1966). The Isla de Lobos and associated reefs, Veracruz, Mexico. Brigham Young Univ. Geol. Stud., 13, 3-46.

Salas-Pérez, J. J., Ocaña-Valencia, A. N. \& González-Gándara, C. (2015). Temperatura superficial del mar y concentración de Clorofila-a en zonas arrecifales y desembocadura de sus ríos en el Golfo de México occidental. En A. GranadosBarba, L. Ortiz-Lozano, D. Salas-Monreal \& C. González-Gándara (Eds.), Aportes al conocimiento del Sistema Arrecifal Veracruzano: hacia el Corredor Arrecifal del Suroeste del Golfo de México (pp. 315-332). México: Universidad Autónoma de Campeche.

Sebens, K. P. \& Miles, J. S. (1988). Sweeper tentacles in a gorgonian octocoral: morphological modifications for interference competition. Biol. Bull. 175, 378-387. http://dx.doi. org/10.2307/1541729

SEMARNAT \& CONANP. (2014). Programa de Manejo Área de Protección de Flora y Fauna, Sistema Arrecifal LobosTuxpan. Recuperado en marzo 07, 2016, disponible en http://www.conanp.gob. $\mathrm{mx} /$ que_hacemos/pdf/programas_manejo/2014/Lobos_tuxpanfinal.pdf

Silva, F. J., Gómez, P. B., Santana, C. E., Silva, M. J., Lima, P. E., Santos, A. M. M. \& Pérez, D. C. (2015). Growth of the tropical zoanthid Palythoa caribaeorum (Cnidaria: Anthozoa) on reefs in northeastern Brazil. An. Acad. Bras. Cienc., 87(2), 1-12. http://dx.doi. org/10.1590/0001-3765201520140475

Suchanek, T. \& Green, D. J. (1981, may). Interspecific competition between Palythoa caribaeorum and other sessile invertebrates on St. Croix Reefs, U.S. Virgin Islands. Proceedings of the Fourth International Coral Reef Symposium, Manila, Philippines, 2, 679-684.

Tunnell, J. W. Jr. (2010). Distribución de los arrecifes. En J. W. Jr. Tunnell, E. A. Chávez \& K. Withers (Eds.), Arrecifes Coralinos del Sur del Golfo de México (pp. 17-29). México: Instituto Politécnico Nacional.

Walther, B. A. \& Moore, J. L. (2005). The concepts of bias, precision and accuracy, and their use in testing the performance of species richness estimators, with a literature review of estimator performance. Ecography, 28, 815-829. http://dx.doi. org/10.1111/j.2005.0906-7590.04112.x 